\title{
Zone Theorem for Arrangements in three dimensions
}

\author{
Sanjeev Saxena* \\ Dept. of Computer Science and Engineering, \\ Indian Institute of Technology, \\ Kanpur, INDIA-208 016
}

June 3, 2020

\begin{abstract}
In this note, a simple description of zone theorem in three dimensions is given.
\end{abstract}

\section{Introduction}

Zone theorem is important in analysing incremental algorithms for constructing arrangements. Most popular text books of Computational Geometry (see e.g. [8, 1]) describe zone theorem for Arrangements in two dimensions. Specialised books like [6, 2] describe zone theorem for hyperplanes in $d$-dimensions. Three dimensional arrangements are useful for constructing higher order Voronoi diagrams in plane [8, 7, 2, 6, 3]. Proofs of Zone theorem in higher dimensions use Euler's relation: $\sum_{i=0}^{d}(-1)^{i} F_{i} \geq 0$ 6, 5, 4]. As most students of Computational Geometry are not familiar with these result, only zone theorem in two dimensions is taught in most Computational Geometry courses. In this note, a proof of zone theorem in three dimensions which can be easily taught in Computational Geometry courses is described. The proof uses zone theorem in two dimensions [8, 1, 6, 4. The proof is a essentially a simplified version of proof given by Edelsbrunner, Seidel and Sharir [4.

\section{Definitions and basic properties}

Arrangement in two dimensions is basically a set of $n$ lines (infinite lines and not segments), and in three dimensions of $n$ planes. We will assume that lines in two dimensions and planes in three dimensions are in general position. Thus,

*E-mail: ssax@iitk.ac.in 
in two dimension no two lines are parallel and no three lines meet in a single point [8, 1, 4. Similarly, in three dimensions we will assume that

- No two planes are parallel. Thus, each pair of planes meet in a line. And any three planes in a point.

- No three planes intersect in a common line and no four planes in a (common) point.

Set of lines, in two dimensions, will partition the plane into regions called faces. And set of planes in three dimensions will partition the space into regions, which we will call cells.

Let $C$ be any bounded cell. As planes are in general position, each point or vertex $v$ in arrangement is determined by three planes. Thus, there will be three edges of $C$ incident at any vertex $v$ of $C$. Moreover, each edge of $C$ is determined by two vertices of $C$. If $\left|V_{C}\right|$ is the number of vertices of $C$ and $\left|E_{C}\right|$ is the number of edges of $C$, then $2\left|E_{C}\right|=3\left|V_{C}\right|$.

As $C$ is on one side of each plane, $C$ will be a convex polytope (3-dimensional analogue of polygon). The set of edges, vertices and faces on boundary of $C$ will form a planar graph. In a planar graph, if $|V|$ is the number of vertices, $|E|$ the number of edges and $|F|$ the number of faces, then by Euler's formula $|E|-|V|+2=|F|$. If $\left|F_{C}\right|$ is the number of faces of $C$ then $\left|F_{C}\right|=\left|E_{C}\right|-$ $\left|V_{C}\right|+2=\left|E_{C}\right|-\frac{2}{3}\left|E_{C}\right|+2=\frac{1}{3}\left|E_{C}\right|+2$. Thus, $\left|E_{C}\right|<3\left|F_{C}\right|$ and hence $\left|V_{C}\right|=\frac{2}{3}\left|E_{C}\right|<2\left|F_{C}\right|$. Or, $\left|V_{C}\right|=O\left(\left|F_{C}\right|\right)$ and $\left|E_{C}\right|=O\left(\left|F_{C}\right|\right)$.

In two dimensions, let $S$ be a line different from $n$ given lines (also in general position). Then $S$ will intersect (cut) some faces of the arrangement. Zone $(S)$ is defined as the set of faces through which line $S$ passes. If $C \in$ zone $(S)$, is a face which is cut, then let $|C|$ be the number of edges on the boundary of face $C$ in the (original) arrangement, then the size of zone $(S),|\operatorname{zone}(S)|=\sum_{C \in \operatorname{zone}(S)}|C|$. It is known that $|\operatorname{zone}(S)|=O(n)([8,1,6,4)$.

Similarly, in three dimensions, let $S$ be a plane different from $n$ given planes (also in general position). Then $S$ will intersect (cut) some cells of the arrangement. Zone $(S)$ is defined as the set of cells which the plane $S$ intersects. If $C \in \operatorname{zone}(S)$, is a cell which is cut, then let $\left|F_{C}\right|$ be the number of faces on the boundary of cell $C$ in the (original) arrangement, then the size of zone $(S)$, $|\operatorname{zone}(S)|=\sum_{C \in \operatorname{Zone}(S)}\left|F_{C}\right|$.

Remark: Normally the size of zone is defined as the sum of number of edges, vertices and faces of all cells in the zone, but as the number of vertices and edges in a cell are $O\left(\left|F_{i}\right|\right)$, the two definitions are equivalent up to multiplicative constants. Moreover, as each face is on boundary of two cells, the total number of cells in a zone will also be bounded by $O\left(\sum_{C}\left|F_{C}\right|\right)$.

\section{Zone Theorem in 3-dimensions}

We will assume that all planes of the arrangement (together with $S$ ) are in general position, as size of zone is not smaller in this case [6, 4, 2, 
Further, let us enclose the arrangement in a "bounding box" [1, 6] by having six planes $x= \pm A, y= \pm A, z= \pm A$ - basically we compute coordinates of all $\left(\begin{array}{l}n \\ 3\end{array}\right)$ vertices of arrangement (by taking every possible set of three planes) and choosing $A$ to be larger than the (absolute value of) largest coordinate. Thus, all cells inside the bounding box will be bounded.

Let $Q$ be any plane of the arrangement. Then as all planes are in general position, each of them will intersect $Q$ in a line. All these lines will lie in the plane $Q$ and form a two-dimensional (planar) arrangement of lines (say $\mathcal{L}_{\mathcal{Q}}$ ).

Let us remove plane $Q$ from the arrangement $\mathcal{A}$ and let the resulting arrangement be called $\mathcal{A}-\mathcal{Q}$.

Let $C$ be a cell in the arrangement $\mathcal{A}$. If the plane $Q$ does not cut (intersect) cell $C$, then cell $C$ and all its faces will be present (unchanged) in arrangement $\mathcal{A}-\mathcal{Q}$.

If the plane $Q$ cuts (intersects) cell $C$, then the cell $C$ gets divided into two parts - say the part of $C$ above the plane $Q$ and the part of $C$ below $Q$ (or if $Q$ is horizontal then left and right of $Q$ ). Let us call the two parts as $C_{1}$ and $C_{2}$. Part of $C$ intersected by $Q$ will lie in plane $Q$ (definition of intersection) and hence will be a face (say $f^{*}$ ) in the two dimensional arrangement $\mathcal{L}_{\mathcal{Q}}$.

If face $f$ is not intersected by $Q$, then face $f$ will be present (unchanged) in either $C_{1}$ or $C_{2}$.

If face $f$ is intersected by $Q$, then $f$ will get split into two parts one above $Q$ and the other below $Q$ (or one on left and the other on right). Let the part in $C_{1}$ be called $f_{1}$ and part in $C_{2}$ be called $f_{2}$. Let the boundary (part common to both) be called $e^{\prime}$. As $e^{\prime}$ is (also) in plane $Q$, $e^{\prime}$ will be an edge in two dimensional arrangement $\mathcal{L}_{\mathcal{Q}}$. Edge $e^{\prime}$ is in face $f^{*}$.

To prove the zone-theorem we need following intermediate result

Theorem 1. Assume $\mathcal{A}$ is an arrangement of $n$ planes, $Q$ is a plane in $\mathcal{A}$, and $S$ is a plane not in $\mathcal{A}$. Let $C$ be a cell in zone $(S)$. Let $f$ be a face of cell $C$ not lying in plane $Q$. Then total number of such pairs $(f, C)$ (of face $f$ and cell $C$ ) is at most the sum of

1. size of zone $(S)$ in arrangement $\mathcal{A}-\mathcal{Q}$ and

2. size of zone $(S)$ in two dimensional arrangement $\mathcal{L}_{\mathcal{Q}}$

Remark: The first size is count of faces (along with their multiplicities) and second of edges (along with their multiplicities).

Proof. Assume that cell $C$ is in zone $(S)$ (of arrangement $\mathcal{A}-\mathcal{Q}$ ) and $f$ is a face of $C$, not lying in (part of) plane $Q$. As $C$ is in zone $(S)$, plane $S$ passes through cell $C$.

If $Q$ does not cut cell $C$, then cell $C$ will be unchanged in arrangement $\mathcal{A}$ (and as $S$ passes through $C$ ), cell $C$ will also be in $\operatorname{zone}(S)$ in arrangement $\mathcal{A}$. In this case $f^{\prime}=f$ and $C^{\prime}=C$.

Let us assume that $Q$ cuts $C$. Then cell $C$ gets divided into two parts (say) $C_{1}$ and $C_{2}$. If the plane $Q$ does not cut face $f$, then face $f$ will remain intact in one part (say) $C_{i}$ (for $i=1$ or 2 ). If part $C_{i}$ contains $f$, then the pair $(f, C)$ 
corresponds to pair $\left(f, C_{i}\right)$. Note that right hand side will be larger if $C_{i}$ is not in the zone (see below).

Since $S$ passes through $C$, it will pass through either $C_{1}$ or $C_{2}$ or both. If $S$ passes through only one part (say) $C_{i}$ (for $i=1$ or 2 ), then only $C_{i}$ will be in the zone $(S)$ in arrangement $\mathcal{A}$. In this case, for pair $(f, C)$ we will have the pair $\left(f, C_{i}\right)$ (or in case $f$ is intersected by $Q$, then pair $\left(f_{i}, C_{i}\right)$ where $f_{i}$ is the part of $f$ in $C_{i}$ ).

We are left with the case when face $f$ is also cut by $Q$ and both $C_{1}$ and $C_{2}$ are in the zone $(S)$.

If $S$ passes through both $C_{1}$ and $C_{2}$, then both $C_{1}$ and $C_{2}$ will be in the zone $(S)$ in arrangement $\mathcal{A}$. As $S$ passes through both $C_{1}$ and $C_{2}$, it will also intersect the common boundary of $C_{1}$ and $C_{2}$. But as $Q$ passes through the common boundary of $C_{1}$ and $C_{2}$, the common part will be a face (say $f^{*}$ ) in the two dimensional arrangement $\mathcal{L}_{\mathcal{Q}}$. And as $S$ intersects $f^{*}$, face $f^{*}$ will be in the two dimensional zone $(S \cap Q)$.

As face $f$ is also cut by $Q$, intersection of $f$ and $Q$ will be a line segment (say) $e^{\prime}$. As $e$ lies in plane $Q, e^{\prime}$ will be an edge in the two dimensional arrangement $\mathcal{L}_{\mathcal{Q}}$. Thus, for the pair $(f, C)$ in three dimensional arrangement, we also have the pair $\left(e^{\prime}, f^{*}\right)$ in the two dimensional arrangement. Thus, there will be two entries which will be counted on the right hand side. On the left hand side we also have two entries $\left(f_{1}, C_{1}\right)$ and $\left(f_{2}, C_{2}\right)$.

Q.E.D.

As each face of a cell in $\mathcal{A}$ is in exactly one plane of the arrangement, it does not lie in remaining $n-1$ planes. Thus, if we take any pair $(f, C)$ (for face $f$ in cell $C$ lying in zone $(S)$ ), it will not lie in $n-1$ planes. Or if we take each plane in turn as plane $Q$ and add we get

$$
(n-1)|\operatorname{zone}(S)| \leq \sum_{Q \in \mathcal{A}}\left(\left|\operatorname{zone}_{\mathcal{A}-\mathcal{Q}}(S)\right|+\left|\operatorname{zone}_{\mathcal{L}_{\mathcal{Q}}}(S \bigcap Q)\right|\right)
$$

To get the bounds, let $z(n)$ be the largest possible value of $|\operatorname{zone}(S)|$ for arrangement of $n$-planes. Then, if we are considering this arrangement and this set $S$ (for which the value of $\mid$ zone $(S) \mid$ is the largest), then we have

$$
(n-1) z(n) \leq \sum_{Q \in \mathcal{A}}\left(\left|\operatorname{zone}_{\mathcal{A}-\mathcal{Q}}(S)\right|+\left|\operatorname{zone}_{\mathcal{L}_{\mathcal{Q}}}(S \bigcap Q)\right|\right)
$$

As $\mathcal{A}-\mathcal{Q}$ is an arrangement of $n-1$ planes ( $Q$ is excluded), $\mid$ zone $_{\mathcal{A}-\mathcal{Q}}(S) \mid \leq$ $z(n-1)$. Further, as two dimensional arrangement $\mathcal{L}_{\mathcal{Q}}$ is in plane $Q$ and each line corresponds to one of the other plane, the number of lines in $\mathcal{L}_{\mathcal{Q}}$ is $n-1$. By the two dimensional zone theorem (see Section 4 or [8, 1, 6, 4), number of edges in zone will be linear. Hence, for some constant $c$, $\mid$ zone $_{\mathcal{L}_{\mathcal{Q}}}(S \cap Q) \mid \leq c(n-1)$. Thus, our equation becomes

$$
(n-1) z(n) \leq \sum_{Q \in \mathcal{A}}(z(n-1)+c(n-1))=n z(n-1)+c n(n-1)
$$


To solve this, we put $f(n)=z(n) / n$ or $z(n)=n f(n)$, the equation becomes

$$
f(n) \leq f(n-1)+c
$$

Or $f(n)=c n$, or $z(n)=n f(n)=c n^{2}$. Hence we get the Zone theorem:

Theorem 2. Assume that we are given an arrangement $\mathcal{A}$ of $n$ planes in three dimension. Let $S$ be a plane different from the planes of the arrangement. Then size of zone $(S)$,

$$
|z o n e(S)|=O\left(n^{2}\right)
$$

\section{Acknowledgements}

Thanks to students of CS663A (2019-2020 batch) for reading an earlier version.

\section{References}

[1] M.de.Berg, M.van Kreveld, M.Overmars and O.Schwarzkops, Computational Geometry, Algorithms and Applications, second edition, 2000, Springer.

[2] H.Edelsbrunner, Algorithms in Combinatorial Geometry, Springer 1987.

[3] H.Edelsbrunner, J.O' Rourke and R.Seidel, Constructing arrangements of lines and hyperplanes with applications, SIAM J. Comput., vol 15, no. 2, pp -, May 1986.

[4] H.Edelsbrunner, R.Seidel and M.Sharir, On the zone theorem for hyperplane arrangements, SIAM J. Comput., vol 22, no. 2, pp 418-429, April 1993.

[5] L.Guibas, Basic Algorithms and Combinatorics in Computational Geometry, 2011.

[6] K.Mulmuley, Computational Geometry, An introduction through randomized algorithms, Prentice Hall, 1994

[7] F.P.Preparata and M.I.Shamos, Computational Geometry, An introduction, 1985, Springer.

[8] J.O' Rourke, Computational Geometry in C, 2nd Ed, Cambridge Univ. Press, 1998. 